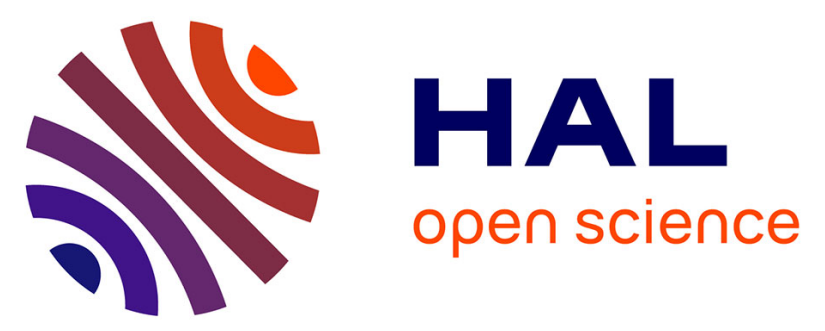

\title{
LES NOYAUX DE TRANSITION 189Ir ET 187Ir SONT-ILS TRIAXIAUX?
}

S. André, J. Boutet, J. Jastrzebski, R. Kaczarowski, J. Lukasiak, J. Rivier, C. Sébille-Schuck, Z. Sujkowski, J. Treherne

\section{- To cite this version:}

S. André, J. Boutet, J. Jastrzebski, R. Kaczarowski, J. Lukasiak, et al.. LES NOYAUX DE TRANSITION 189Ir ET 187Ir SONT-ILS TRIAXIAUX?. Colloquium on Nuclear Surface, 1975, Dijon, France. pp.C5-106-C5-106, 10.1051/jphyscol:1975523 。 jpa-00216378

\section{HAL Id: jpa-00216378 https://hal.science/jpa-00216378}

Submitted on 1 Jan 1975

HAL is a multi-disciplinary open access archive for the deposit and dissemination of scientific research documents, whether they are published or not. The documents may come from teaching and research institutions in France or abroad, or from public or private research centers.
L'archive ouverte pluridisciplinaire HAL, est destinée au dépôt et à la diffusion de documents scientifiques de niveau recherche, publiés ou non, émanant des établissements d'enseignement et de recherche français ou étrangers, des laboratoires publics ou privés. 


\title{
LES NOYAUX DE TRANSITION ${ }^{189}$ Ir ET ${ }^{187}$ Ir SONT-ILS TRIAXIAUX ?
}

S. ANDRÉ $(*)$, J. BOUTET (*), J. JASTRZEBSKI (**), R. KACZAROWSKI $\left({ }^{* *}\right)$, J. LUKASIAK $(* *)$, J. RIVIER, C. SÉBILLE-SCHUCK $\left(^{* * *}\right)$, Z. SUJKOWSKI (**) et J. TREHERNE $\left.{ }^{*}\right)$

\author{
(*) I.S.N. Grenoble
}

$\left({ }^{* *}\right)$ I.B.J. Swierk (Pologne)

$(* * *)$ C.S.N.S.M. Orsay

\begin{abstract}
Résumé. - Les niveaux excités des noyaux ${ }^{189}$ Ir et ${ }^{187}$ Ir ont été étudiés par réaction $(\alpha, 2 \mathrm{n} \gamma)$ sur le faisceau du cyclotron de Grenoble.

Les schémas détaillés qui ont été établis mettent en évidence :

1) des états de parité positive qui s'interprètent comme appartenant aux deux bandes $3 / 2^{+}|402|$ (fondamental) et $1 / 2^{+}|400|$ mélangées par interaction de Coriolis (noyaux à symétrie axiale de déformation positive);

2) une bande découplée construite sur un état $9 / 2^{-}$(h 9/2) décrite de manière équivalente par un modèle à symétrie axiale et déformation positive ou par un modèle à particule-plus-rotor asymétrique [1];

3) une structure complexe pour les niveaux de parité négative associés à l'isomère $11 / 2^{-}(\mathrm{h} 11 / 2)$. Ce système est correctement prédit par le modèle à rotor asymétrique [1].
\end{abstract}

\begin{abstract}
Excited levels in ${ }^{189} \mathrm{Ir}$ and ${ }^{187} \mathrm{Ir}$ have been studied by in beam $\gamma$-ray spectroscopy at the Grenoble isochronous Cyclotron. The detailed level schemes deduced show :

1) Positive parity states which may be interpreted as belonging to two bands $3 / 2^{+}[402]$ (g.s.) and $1 / 2^{+}[400]$ mixed by the Coriolis interaction (axially symmetric nucleus with positive deformation).

2) A rotation aligned band built on the $9 / 2^{-}\left(\mathrm{h}_{9 / 2}\right)$ state described either by a particle + symmetric rotor model (with positive deformation) or by a particle + asymmetric rotor [1].

3) A negative parity levels of a complex structure associated with the $11 / 2^{-}\left(\mathrm{h}_{11 / 2}\right)$ isomer and predicted correctly by the asymmetric rotor model [1].
\end{abstract}

\section{Bibliographie}

[1] MEYER ter Vehn, J., LBL, Report 3459, janvier 1975.

Article à paraître dans Nuclear' Physics. 